\title{
UCRL-PROC-207943
}

LAWRENCE LIVERMORE N A T IO N A L LABORATORY

\section{The Nature of Emission from Optical Breakdown Induced by Pulses of fs and ns Duration}

C. W. Carr, M. D. Feit, A. M. Rubenchik, P. Demange, S. Kucheyev, M. D. Shirk, H. B. Radousky, S. G. Demos

November 11, 2004

Boulder Damage Symposium XXXVI Annual Symposium Boulder, CO, United States September 20, 2004 through September 22, 2004 
This document was prepared as an account of work sponsored by an agency of the United States Government. Neither the United States Government nor the University of California nor any of their employees, makes any warranty, express or implied, or assumes any legal liability or responsibility for the accuracy, completeness, or usefulness of any information, apparatus, product, or process disclosed, or represents that its use would not infringe privately owned rights. Reference herein to any specific commercial product, process, or service by trade name, trademark, manufacturer, or otherwise, does not necessarily constitute or imply its endorsement, recommendation, or favoring by the United States Government or the University of California. The views and opinions of authors expressed herein do not necessarily state or reflect those of the United States Government or the University of California, and shall not be used for advertising or product endorsement purposes. 


\title{
The Nature of Emission from Optical Breakdown Induced by Pulses of fs and ns Duration
}

\author{
H. B. Radousky, and S. G. Demos \\ Lawrence Livermore National Laboratory \\ 7000 East Avenue, L-592 \\ Livermore, CA 94550
}

C. W. Carr", M. D. Feit, A. M. Rubenchik, P. Demange, S. Kucheyev, M. D. Shirk,

\begin{abstract}
Spectral emission from optical breakdown in the bulk of a transparent dielectric contains information about the nature of the breakdown medium. We have made time resolved measurements of the breakdown induced emission caused by nanosecond and femtosecond infrared laser pulses. We previously demonstrated that the emission due to ns pulses is blackbody in nature allowing determination of the fireball temperature and pressure during and after the damage event. The emission due to femtosecond pulse breakdown is not blackbody in nature; two different spectral distributions being noted. In one case, the peak spectral distribution occurs at the second harmonic of the incident radiation, in the other the distribution is broader and flatter and presumably due to continuum generation. The differences between ns and fs breakdown emission can be explained by the differing breakdown region geometries for the two pulse durations. The possibility to use spectral emission as a diagnostic of the emission region morphology will be discussed.

Keywords: Laser-induced-damage, KDP, DKDP, emission
\end{abstract}

\section{INTRODUCTION}

Recently, we demonstrated that breakdown initiated with ns irradiation can produce an ionized region sufficiently large and dense enough to thermalize the plasma emission (every photon will be scattered multiple times) ${ }^{1}$. The temporal evolution of this thermalized radiation gave information about plasma temperatures, local pressures, electron densities, and energy dissipation mechanisms. In the present work, we use the same method to evaluate the radiation produced by fs pulses in LiF, fused silica $\left(\mathrm{SiO}_{2}\right)$, and DKDP $\left(\mathrm{KD}_{2} \mathrm{PO}_{4}\right)$. The broadband spectra are compared to, and found to be fundamentally different from those obtained in the same materials when nanosecond pulses initiate the breakdown. In addition, the spectra produced by fs radiation are shown to fall into two distinct categories that correspond to different breakdown morphologies.

\section{EXPERIMENTAL}

A Ti:Saphire laser with a pulse duration of $150 \mathrm{fs}$ operating at $820 \mathrm{~nm}$ with a measured bandwidth of $\sim 10 \mathrm{~nm}$ was one of the two lasers used in these experiments. A mechanical shutter isolated single pulses from the output $100 \mathrm{~Hz}$ pulse train. The power of the laser pulse was $1.5+/-0.3 \mathrm{MW}$ and the peak fluence at focus was on the order of $3+/-0.6 \mathrm{~J} / \mathrm{cm}^{2}$. The $20 \%$ energy / fluence fluctuations resulted from running the laser at such a (relatively) low repetition rate. A Q-switched ND:Yag was also used to provide 5-ns pulses of 1064nm light.

\footnotetext{
* Correspondence: 925 422-8755, carr19@1lnl.gov
} 
A 3-inch focal length off-axis parabolic mirror was used to focus the laser to a $10-\mu \mathrm{m}$ to $30-\mu \mathrm{m}$ spot (depending on wavelength) in the bulk of a sample. Light originating from the geometric focus of the off-axis parabolic mirror was collected, transported, and focused by reflective optics onto the slit of a spectrometer (as seen in figure 1). An intensified charged coupled device (ICCD) allowed the emission to be gated with 5-ns temporal resolution. This allowed the emission from ns-pulses to be studied at various delay times and avoided the collection of long term (ms) thermal emission when damage is produced by fs-pulses, as seen in previous work ${ }^{2}$. The response of the system was calibrated with a combination of black body and $\mathrm{D}_{2}$ light sources.

Fig 1. A schematic representation of the apparatus.

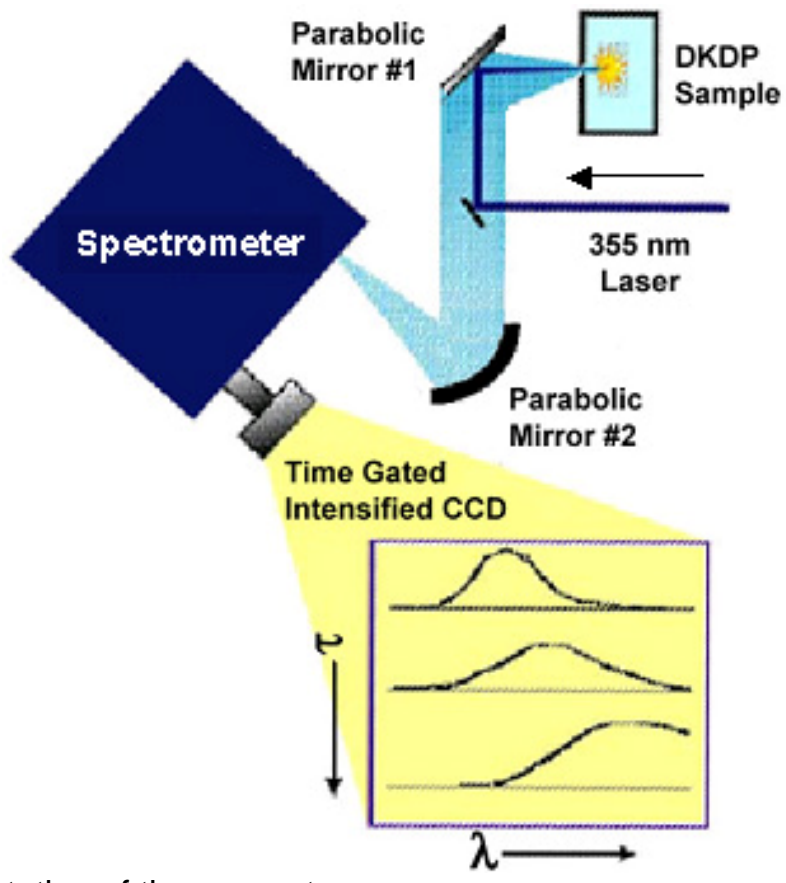

Data were collected over a spectral range of $250 \mathrm{~nm}$ to $550 \mathrm{~nm}$ for $\mathrm{LiF}, \mathrm{SiO}_{2}$, and DKDP and may be seen in figures 2 and 3. Emission observed from ns and fs damage differed dramatically in both duration and spectral content. For damage induced with ns-radiation emission persisted into the microsecond regime ${ }^{1}$. In contrast, we were able to detect signal only when the 5 -ns integration window contained the 0 ns delay point. Such a signal for a $\mathrm{SiO}_{2}$ sample is shown in figure 2 and compared to breakdown spectra for delay of $2 \mathrm{~ns}$ and $8 \mathrm{~ns}$ under ns-irradiation (delay times were measured from the center of the 5-ns integration window). 


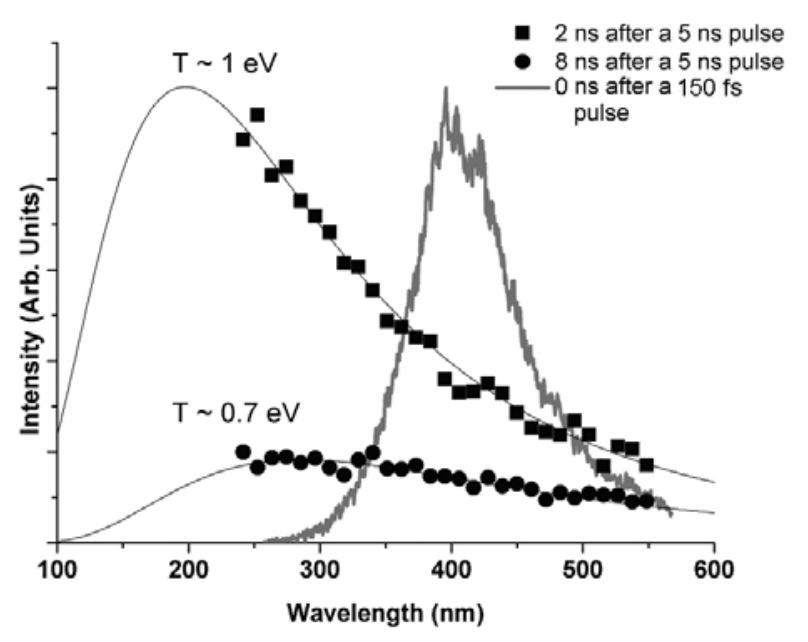

Fig 2. The solid points are spectral emission from experiments using a 5-ns 1064-nm laser focused to a 30 $\mu \mathrm{m}$ spot in the bulk of $\mathrm{SiO}_{2}$. The thin lines through the points are fits to the Planckian distribution. The approximate temperatures indicated by each spectra is stated. For comparison, the thick gray line is the emission produced in $\mathrm{SiO}_{2}$ when the 820-nm 150-fs pulse is used. In each case, signal is obtained over a 5ns temporal window with delay times measured from the center of the window.

The spectra obtained from DKDP, $\mathrm{SiO}_{2}$ and $\mathrm{LiF}$ under 150 fs radiation may be seen in figure 3. Emission from $\mathrm{SiO}_{2}$ and $\mathrm{LiF}$ appear to have similar spectral profiles. Both materials have spectra that peaked at the second harmonic of the laser line with a tail in the red. However, the spectrum from DKDP is much broader and lacks the distinctive peak seen in the spectra obtained from $\mathrm{SiO}_{2}$ and $\mathrm{LiF}$. This difference in the spectra indicate a material dependence to the breakdown mechanism.

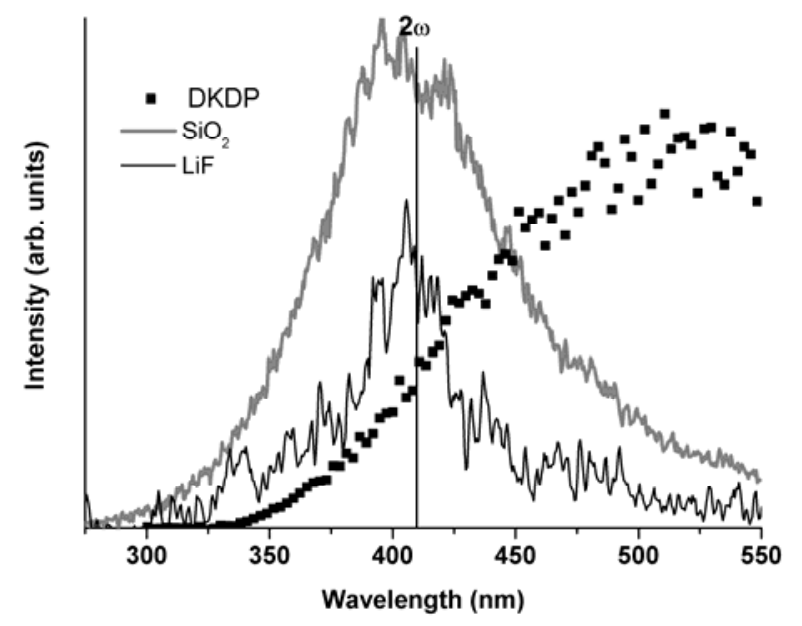

Fig 3. The spectra from DKDP, $\mathrm{SiO}_{2}$ and $\mathrm{LiF}$ under 150-fs radiation. The vertical line denotes $410 \mathrm{~nm}$ (twice the frequency of the laser line).

\section{DISCUSSION}

To understand the mechanisms involved we first consider the source of each type of spectra. The peak in LiF and $\mathrm{SiO}_{2}$ at $410 \mathrm{~nm}$ can be attributed to second harmonic generation (SHG) from the $820 \mathrm{~nm}$ laser line. We postulate this second harmonic generation is plasma induced, a well known phenomenon ${ }^{3}$ for critical density plasmas. 
The spectral profile obtained from DKDP (see figure 3) did not exhibit the SHG peak at $410 \mathrm{~nm}$. The origin of both the DKDP and $\mathrm{SiO}_{2} / \mathrm{LiF}$ type spectra can be understood by considering the effects of self-focusing. Self-focusing occurs when the power of the laser $(P)$ exceeds a material-dependent critical power $\left(P_{c r}\right)$, given by $P_{c r}=3.77 \lambda_{0}^{2} / 8 \pi n_{0} n_{2}$ where $\lambda$ is the free space wavelength of the laser light and $n_{0}$ and $n_{2}$ are the linear and nonlinear indices of refraction, respectively. Various parameters of the materials as well as the calculated critical power of each can be found in table 1.

\begin{tabular}{|c|c|c|c|r|}
\hline Sample & $\mathrm{Eg}(\mathrm{eV})$ & $\mathrm{n}_{0}$ & $\begin{array}{c}\mathrm{n}_{2}\left(\mathrm{~cm}^{2} \mathrm{~W}^{-1}\right) \\
\times 10^{-16}\end{array}$ & $\mathrm{P}_{\mathrm{cr}}(\mathrm{MW})$ \\
\hline $\mathrm{LiF}$ & $13.7^{4}$ & $1.3^{5}$ & $0.67^{5}$ & 11.3 \\
\hline $\mathrm{DKDP}$ & $8.4^{6}$ & -1.5 & $10.2^{7}$ & 0.64 \\
\hline $\mathrm{SiO}_{2}$ & $7.8^{5}$ & $1.5^{8}$ & $2.4^{8}$ & 2.7 \\
\hline
\end{tabular}

Table 1. Select physical properties of the samples used in these experiments.

By comparing the power of the laser pulse $(1.5+/-0.3 \mathrm{MW})$, and the critical power of each of the materials studied, a correlation between spectra type and critical power is demonstarted. In $\mathrm{SiO}_{2}$ and $\mathrm{LiF}$ (where the second harmonic spectrum is always present) the laser power used in this experiment was not above the critical power needed for self-focusing. In DKDP, however, the laser power is well above $\mathrm{P}_{\mathrm{cr}}$. By definition, self-focusing and filamentation occur when the power of the laser exceeds the $P_{c r}$ of the material. In our experiments, self-focusing will not play a role in breakdown dynamics in $\mathrm{SiO}_{2}$ and $\mathrm{LiF}$, but will be present in DKDP. This leads to the observation of second harmonic generation in $\mathrm{SiO}_{2}$ and $\mathrm{LiF}$, but not in DKDP as explained below.

The dependence of the emission spectra on critical power, pulse power, and pulse duration can be understood by considering how the geometry of the ionized region is affected by these parameters. For nanosecond pulses the emission spectra have been shown to be immutable over a wide range of pulse energies and laser wavelengths ${ }^{1}$. These results are readily attainable for ns irradiation at powers below those necessary for self-focusing. During breakdown produced by nanosecond pulses in this regime, a radiation driven ionization wave propagates back towards the laser from the point of initial breakdown, producing a spherical or elliptical volume of plasma (see figure 4). Under proper conditions, the emission from this plasma is self-trapped and observed as thermal radiation which may be used to determine the temperature of the plasma and lattice. ${ }^{1}$

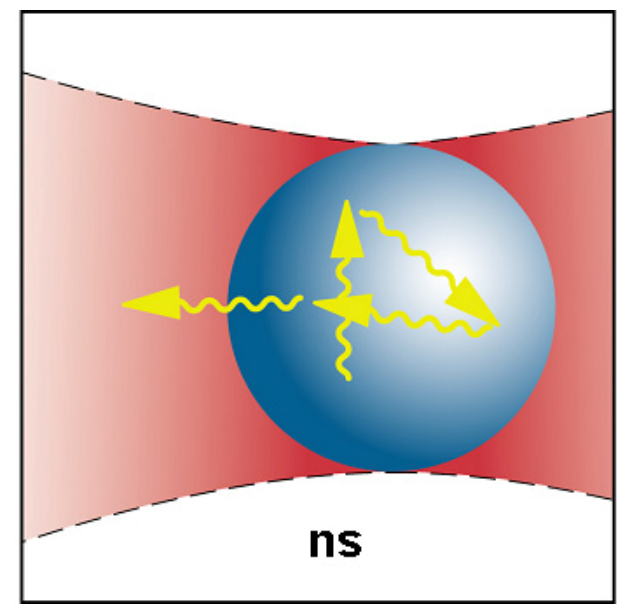

Fig 4. During ns-induced damage a spherical (or ellipsoid) region of plasma is formed which is significantly large enough to thermalize the radiation leaving the area.

Damage produced by a femtosecond pulse can assume two distinct morphologies depending on the power of the pulse and the focal geometry (see figure 5$)^{9}$. For femtosecond laser pulses below the critical power, the initial breakdown is manifested as an ionized region in the shape of a disk less than a micron in thickness ${ }^{10}$. 
The electron density in this region reaches the critical density for the pump wavelength very quickly (compared to the duration of the pulse) ${ }^{11}$. The high electron density allows the pump radiation to penetrate only a short distance (the plasma skin depth), restricting expansion of the ionized region away from the site of initial breakdown by reflecting and scattering the incident laser light. Unlike the case of nanosecond duration, the ionization waves for femtosecond pulses (which propagate at velocities on the order of the sound speed in the material) have insufficient time to expand the ionized region which remains a thin disk ${ }^{12}$.

Second harmonic generation of light from the interaction of laser pulses with plasma in the backscattering geometry is a well-known phenomenon ${ }^{9}$ near the critical density points. Incident laser energy is transformed into plasma waves due to linear or non-linear processes. The second harmonic radiation is produced by coupling of two plasma waves or plasma and electromagnetic waves and is often accompanied by spectral broadening from plasma turbulence ${ }^{3}$. It should be noted that this is a fundamentally different process than phase matching in non-linear media; SHG radiation is backscattered and is not dependent on material orientation.

We believe the observed broadening has a dynamic nature associated with rapid change in the electron density. It is shown previously ${ }^{11}$ that the time scale for the increase in electron density from very low or no background density to critical density $n_{c}$ is on the order of tens of fs. The increase in electron density gives rise to a rapid change in the index of refraction of the ionized region, and a high velocity ionization front both of which can contribute to the observed broadening. It is also important that for effective SHG the critical surface must be locally flat.
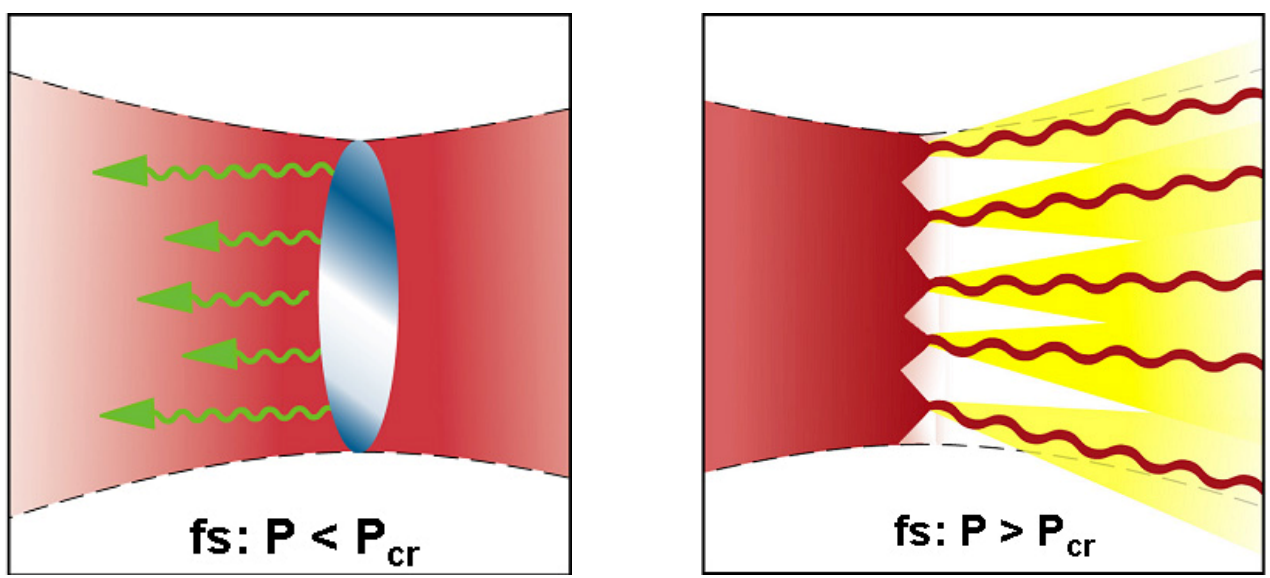

Fig 5. Left, femtosecond radiation below critical power produces a thin disk of plasma which second harmonic light can be generated by reflecting off the expanding, turbulent ionization front. Right, filimentation and super-continuum generated when femtosecond radiation is above the critical power of the material.

When the laser pulse is above the critical power, self focusing and filamentation occur before the focus is reached and the breakdown morphology changes from a disk of high electron density plasma to filaments of relatively low electron density ${ }^{13}$. Each filament contains on the order of one critical power. The lower electron densities associated with filamentation do not allow light to excite plasma waves effectively, precluding second harmonic generation. However, this breakdown geometry, due to the interplay between self-focusing and multi-photon ionization, can result in white light continuum generation ${ }^{14,15}$. As the power of the laser pulses was twice $P_{c r}$ for DKDP, filamentation, and therefore supercontinuum generation should be expected in DKDP. 


\section{CONCLUSION}

In conclusion, three fundamentally different types of emission were observed for nanosecond-induced breakdown, femtosecond-induced breakdown with $\mathrm{P}<\mathrm{Pcr}$, and femtosecond-induced breakdown with $\mathrm{P}>$ Pcr. Each emission type corresponds to different breakdown morphology and hence the breakdown morphology can be determined by monitoring the accompanying emission.

\section{REFERENCES}

C. W. Carr, H. B. Radousky, A. M. Rubenchik, M. D. Feit, and S. G. Demos, Localized dynamics during laser-induced damage in optical materials,Physical Review Letters 92, 087401 (2004). A. V. Gorbunov and M. Maksimuk, Kinetics of local optical breakdown in the volume of alkalihalide crystals. I. Development of a cavity,Fizika Tverdogo Tela 36, 1416 (1994).

3 N. G. Basov, V. Y. Bychenkov, O. N. Krokhin, M. V. Osipov, A. A. Rupasov, V. P. Silin, G. V. Sklizkov, A. N. Starodub, V. T. Tikhonchuk, and A. S. Shikanov, 2nd Harmonic-Generation in the Laser Plasma,Kvantovaya Elektronika 6, 1829 (1979). M. Fox, Optical Properties of Solids (Oxford University Press, 2001).

R. Desalvo, A. A. Said, D. J. Hagan, E. W. Vanstryland, and M. Sheikbahae, Infrared to Ultraviolet Measurements of Two-Photon Absorption and N(2) in Wide Bandgap Solids,IEEE Journal of Quantum Electronics 32, 1324 (1996). I. N. Ogorodnikov, V. A. Pustovarov, B. V. Shul'gin, V. T. Kuanyshev, and M. K. Satybaldieva, Low-temperature time-resolved vacuum ultraviolet luminescent spectroscopy of KH/sub 2/PO/sub 4/ crystals with defects, Optika i Spektroskopiya 91, 243 (2001).

$7 \quad$ W. L. Smith, J. H. Bechtel, and N. Bloembergen, Dielectric-Breakdown Threshold and NonlinearRefractive-Index Measurements with Picosecond Laser Pulses,Physical Review B 12, 706 (1975). W. T. White, W. L. Smith, and D. Milam, Direct measurement of the nonlinear refractive-index coefficient (gamma) at $355 \mathrm{~nm}$ in fused silica and in BK-10 glass,Optics Letters 9, 10 (1984). N. T. Nguyen, A. Saliminia, W. Liu, S. L. Chin, and R. Vallee, Optical breakdown versus filamentation in fused silica by use of femtosecond infrared laser pulses, Optics Letters 28, 1591 (2003).

10 M. D. Feit, A. M. Komashko, and A. M. Rubenchik, Ultra-short pulse laser interation with transparent dielectrics,Applied Physics A 79, 1657 (2004).

11 B. C. Stuart, M. D. Feit, S. Herman, A. M. Rubenchik, B. W. Shore, and M. D. Perry, Nanosecondto-femtosecond laser-induced breakdown in dielectrics,Physical Review B (Condensed Matter) 53, 1749 (1996).

12 Y. P. Raizer, Gas Discharge Physics (Springer-Verlag, New York, 1991).

13 P. Sprangle, J. R. Penano, and B. Hafizi, Propagation of intense short laser pulses in the atmosphere,Physical Review E 66, 046418 (2002).

14 A. Brodeur and S. L. Chin, Ultrafast white-light continuum generation and self-focusing in transparent condensed media,Journal of the Optical Society of America B-Optical Physics 16, 637 (1999).

15 R. R. Alfano,The Supercontinuum Laser Source, (Springer-Verlag, New York, 1989). 